\title{
ESTUDIO COMPARATIVO ENTRE FLUORESCENCIA DE RAYOS-X Y REFLECTANCIA DIFUSA DE INFRARROJOS CERCANOS PARA LA DETERMINACIÓN DE LA RETENCIÓN EN MADERA IMPREGNADA CON ARSENIATO DE COBRE CROMATADO
}

\author{
COMPARATIVE STUDY BETWEEN X-RAY FLUORESCENCE AND NEAR \\ INFRARED DIFFUSE REFLECTANCE FOR THE DETERMINATION OF \\ RETENTION IN WOOD IMPREGNATED WITH CHROMATED COPPER \\ ARSENATE
}

\author{
Ricardo Baettig-Palma ${ }^{1, \wedge}$, Jorge Cornejo-Troncoso ${ }^{1,2}$, Marina Salas-Maureira ${ }^{1}$, \\ Jaime Tapia-Sanhueza ${ }^{2}$
}

\begin{abstract}
RESUMEN
Se realizó un análisis comparativo de la precisión de los resultados en la determinación de la retención de óxidos de cobre, cromo y arsénico en madera impregnada con arseniato de cobre cromatado entre fluorescencia de rayos X y espectrometría de reflectancia difusa de infrarrojos cercanos. Los resultados de la calidad de las predicciones fueron comparados entre sí y con valores de retención obtenidos experimentalmente por método gravimétrico, absorción atómica y por cenizas resultantes de su calcinación. Los resultados del coeficiente de determinación del análisis de regresión entre la intensidad de fluorescencia de rayos X de las líneas K-Alpha y entre el espectro de reflectancia difusa de infrarrojos con respecto a la retención medida por métodos convencionales se encontraron en un rango de 0,909 a 0,986. Los resultados obtenidos pueden ser considerados aceptables para fines predictivos o incluso adecuados para propósitos de control de calidad. A su vez, al ser comparados por sus niveles de error de estimación, estos resultados fueron mejores que los obtenidos por otros estudios similares.
\end{abstract}

Palabras clave: Absorción atómica, arseniato de cobre cromatado, fluorescencia de rayos X, madera impregnada, reflectancia difusa infrarroja.

\begin{abstract}
This paper evaluated the precision of predictions for chromated copper arsenate preservative retention in wood using X-ray fluorescence and near infrared diffuse reflectance. The quality of the predictions were compared with each other and with retention values obtained experimentally by gravimetric method, atomic absorption and total ash content. Coefficients of determination between the K-Alpha fluorescence intensities and near infrared diffuse reflectance with regard to conventional methods were between 0,909 and 0,986. The results can be considered acceptable for predictive purposes or even good for quality control purposes. In turn, the prediction errors were better than obtained by several studies.
\end{abstract}

Keywords: Atomic absorption, chromated copper arsenate, near-infrared diffuse reflectance, treated wood, X-ray fluorescence.

\footnotetext{
${ }^{1}$ Centro Tecnológico del Álamo. Facultad de Ciencias Forestales. Universidad de Talca. Casilla 747 - Talca - Chile.

${ }^{2}$ Instituto de Química de Recursos Naturales, Universidad de Talca.

^Corresponding author : rbaettig@utalca.cl

Received: 22.10.2013 Accepted: 24.04. 2014
} 


\section{INTRODUCCIÓN}

Para alargar la vida útil de la madera en contacto o hincada en el suelo, como es el caso de postes, polines de uso agrícola y fundaciones de pilotes de madera, la impregnación con preservante arseniato de cobre cromatado (CCA) ha demostrado ser eficaz. El preservante CCA es una mezcla de sales metálicas hidrosolubles, cuya composición se expresa en óxidos activos de cobre, cromo y arsénico. La más utilizada actualmente es CCA-C que se comercializa como sal libre en solución concentrada cuya composición varía entre $60 \%$ y $70 \%$ de preservante como óxidos activos. De acuerdo a la norma AWPA P5 (AWPA 1991) este producto comercial contiene $47,5 \%$ de óxido de cromo (III) $\left(\mathrm{CrO}_{3}\right), 18,5 \%$ de óxido de cobre (CuO) y 34,0 $\%$ de peróxido de arsénico $\left(\mathrm{As}_{2} \mathrm{O}_{5}\right)$. El cobre cumple la función de fungicida, el arsénico cumple la función de insecticida y el cromo se relaciona con la fijación de las sales en la estructura de las paredes celulares. La eficacia de impregnación se determina mediante dos indicadores uno cualitativo y otro cuantitativo: la penetración y la retención. La penetración determina la homogeneidad en profundidad que consigue el proceso de impregnación. El método más simple y económico para la determinación de la penetración consiste en la aplicación de soluciones de tinción sobre las superficies expuestas. Se utiliza por lo general una solución de cromazurol, que tiñe la madera de color azul intenso por la presencia de cobre. Se utiliza también solución de 1-(2-Piridilazo)-2-naftol o reactivo PAN, en este caso la madera se torna de color magenta con la presencia de cobre. A su vez, la retención determina de forma cuantitativa la cantidad de óxidos que logran ser fijados en la madera. En Chile los requisitos para la madera impregnada con CCA se encuentran en la norma $\mathrm{NCh}$ 819 Of 2003, que exige que el $90 \%$ de la muestra supere unas retenciones de $4 \mathrm{~kg}$ de óxido activo de CCA por cada $\mathrm{m}^{3}$ de madera para la construcción; $6,4 \mathrm{~kg}$ para polines agrícolas; $9,6 \mathrm{~kg}$ para postes y $40 \mathrm{~kg}$ para construcciones que estarán en contacto con agua de mar. Para maderas cuya densidad es de $400 \mathrm{~kg} \mathrm{~m}^{-3}$ estos valores de retención corresponden a concentraciones de 10000, 15000, 24000 y 100000 ppm en peso de óxidos activos, es decir, en la suma de óxido de cromo, óxido de cobre y peróxido de arsénico. Estas concentraciones son muy altas en relación a las concentraciones de elementos pesados en la madera natural. De acuerdo a Fengel y Wegener (1984) en la madera se encuentran en orden decreciente los elementos calcio, potasio, magnesio, manganeso y fósforo en concentraciones que usualmente van desde 1100 a 30 ppm en masa, junto con otros elementos químicos que se encuentran como sólo como trazas.

A nivel de radiaciones de onda muy corta, como son los rayos $\mathrm{X}$, por medio del efecto fotoeléctrico se puede generar una re-emisión de fotones cuyo perfil energético es característico del elemento químico irradiado, fenómeno conocido como fluorescencia de rayos X (XRF). El análisis espectral de los rayos X reemitidos por fluorescencia permite la obtención de "firmas" características de los distintos elementos químicos presentes en la muestra. Análisis de las intensidades relativas de cada firma permiten obtener concentraciones de elementos (Baettig et al. 2007). La estructura básica de todos los aparatos de fluorescencia de rayos X por dispersión de energía es esencialmente la misma: una fuente de radiación ionizante ya sea monocromática (isótopo radiactivo) o policromática (tubo de rayos X) irradia la muestra y un detector espectrométrico captura una parte de los fotones de fluorescencia re-emitidos. Las diferencias energéticas entre órbitas electrónicas son más importantes a medida que el átomo es más pesado, de modo que los picos empiezan a situarse en gamas de energía cada vez más altas a medida que aumenta la masa atómica de los elementos. Dada las altas concentraciones de cromo, cobre y arsénico en madera tratada con CCA, un experimento de fluorescencia de rayos X (XRF) arroja 6 picos espectrales claramente definidos que corresponden a las 3 líneas K-Alpha $(\mathrm{K} \alpha)$ a $5,41 \mathrm{keV}$ para cromo, $8,05 \mathrm{keV}$ para cobre y $10,54 \mathrm{keV}$ para arsénico, respectivamente, y otros 3 picos menos intensos correspondientes a las líneas K-Beta $(\mathrm{K} \beta)(5,95 \mathrm{keV}$ para cromo, $8,90 \mathrm{keV}$ para cobre y $11,73 \mathrm{keV}$ para arsénico, respectivamente).

El método de espectrometría de reflectancia difusa de infrarrojos cercanos (NIRS) se ha visualizado como una técnica rápida y de fácil implementación para la determinación de la retención de CCA (So et al. 2004). La banda infrarroja cercana es considerada como la zona abarcando un rango espectral entre 780 y $2500 \mathrm{~nm}$ (Osborne 2006). La técnica de espectroscopía infrarroja más a menudo utilizada consiste en recolectar la reflexión difusa de un haz incidiendo en una muestra previamente homogeneizada mediante la obtención de polvo seco y comprimido. Las mediciones se realizan con una alta rapidez operacional, pudiéndose llegar 
comúnmente a una resolución espectral de $1 \mathrm{~nm}$. Las señales de reflectancia registradas son producidas por vibraciones longitudinales y torsionales en los enlaces entre los átomos de carbono, nitrógeno, hidrógeno, oxígeno, fósforo y azufre, y proveen información acerca de la proporción de cada elemento en el material analizado (Brunet et al. 2007). Los principales enlaces involucrados corresponden a C-H, N-H y O-H (Osborne 2006). Ahora bien, los espectros en el infrarrojo cercano son difíciles de interpretar en forma directa porque surgen de la superposición de sobretonos débiles con las bandas vibracionales fundamentales (Chang et al. 2001). Por otra parte, el espectro infrarrojo es influenciado por la estructura física de la muestra, determinada por el tamaño, forma y ordenamiento de las partículas, los espacios vacíos entre ellas, etc. (Osborne 2006). Para reducir este problema con frecuencia los datos son pretratados antes de entrar a un análisis multivariado por medio de la obtención de la primera derivada de la reflectancia, calculada como la diferencia de intensidades entre dos longitudes de onda adyacentes. Aunque hay otros métodos que también se utilizan para reducir este efecto, como la corrección multiplicativa de la dispersión (MSC) y la variación normal estandarizada (SNV) (Osborne 2006).

Básicamente la espectrofotometría de absorción atómica (AA) consiste en medir absorción de energía de un haz de radiación electromagnética en el rango ultravioleta-visible transmitido a través de la muestra previamente atomizada generalmente por una llama, de modo que los electrones de los átomos pueden ser promovidos a orbitales más altos por un instante mediante la absorción de una cantidad específica de energía proporcionada por el haz incidente (Skoog et al. 2001). Como estas transiciones son características de un elemento particular, y en general, como cada longitud de onda corresponde a un único elemento químico, a partir del haz transmitido y usando la ecuación de Beer-Lambert es factible calcular cuántas de estas transiciones tienen lugar, y así obtener una señal que es proporcional a la concentración del elemento que se mide (Welz et al. 1999). Sin embargo, la mayor dificultad que presenta la AA frente a los métodos XRF y NIRS para la determinación de metales en maderas impregnadas, es que necesariamente la muestra requiere un riguroso tratamiento químico (digestión) previo a la cuantificación del elemento en el espectrofotómetro, por lo que la transforma en una metodología lenta para este tipo de matriz (Gaspar et al. 2009, Hein et al. 2011, Krishna et al. 2012, Qi et al. 2013).

Pese a que el análisis de la retención de madera impregnada por XRF está normalizado de acuerdo a la norma AWPAA9 (1990), han sido publicados pocos estudios referidos a la obtención de curvas de calibración de XRF para la madera tratada con preservantes. Block et al. (2007) realizaron la evaluación de la capacidad de dos dispositivos comerciales portátiles de XRF para medir la concentración de arsénico en madera tratada con CCA. Expresando la repetibilidad del equipo como 3 veces su coeficiente de variación, llegaron a valores inferiores a $8 \%$ y $3 \%$ en muestras de aserrín impregnado con concentraciones de arsénico superiores a 2000 $\mathrm{mg} \mathrm{kg}^{-1}$ usando dos dispositivos distintos. Más recientemente, Zielenkiewicz et al. (2012) han propuesto un método de calibración de un dispositivo de XRF para la medición de la retención en madera impregnada con preservante cobre cuaternario alcalino (ACQ). Este compuesto consiste en $66 \%$ de óxido de cobre y $33 \%$ de cloruro de didecildimetil amonio cuaternario. Para ello irradiaron distintas superficies de cuatro muestras sólidas de madera impregnada. Utilizaron la intensidad de radiación fluorescente $\mathrm{K} \alpha$ de cobre $(8,05 \mathrm{keV})$ como un estimador de la concentración de óxido de cobre en un total de 23 determinaciones obteniendo un valor $\mathrm{R}^{2}$ de 0,897. So et al. (2004) utilizaron un dispositivo comercial NIRS para determinar la retención de preservantes en la madera usando regresión de mínimos cuadrados parciales (PLSR). Los preservantes estudiados fueron arseniato de cobre cromatado, cobre cuaternario alcalino y amoniacal arseniato de zinc y cobre (50\% óxido de cobre, $25 \%$ óxido de zinc y $25 \%$ pentóxido de arsénico). Los valores $\mathrm{R}^{2}$ en el conjunto de calibración llegaron a 0,86, 0,93 y 0,97 para las concentraciones de pentóxido de arsénico, óxido de cromo (III) y óxido de cobre, respectivamente. En otro estudio semejante So et al. (2006) utilizaron un dispositivo NIR prototipo para determinar la retención de óxido de cobre en muestras sólidas de madera impregnadas con cobre cuaternario alcalino, obteniendo un valor $\mathrm{R}^{2}$ de 0,94 para el total de 14 datos. Taylor y Lloyd (2007) en madera impregnada con octoborato de sodio determinaron un valor $\mathrm{R}^{2}$ de 0,96 en el conjunto de 18 datos de calibración, que posteriormente se redujo a 0,86 en el conjunto de predicción conformado por otras 10 muestras diferentes. Tomando como base estos resultados bibliográficos, este estudio tiene por objetivo evaluar comparativamente los resultados experimentales en la capacidad predictiva para la determinación de la retención de arseniato 
de cobre cromatado entre un dispositivo prototipo XRF y un dispositivo NIR de uso comercial. Se evalúa la robustez de la capacidad predictiva de la retención de óxidos en madera impregnada con CCA-C mediante distintos tipos de análisis de regresión y los resultados de estas predicciones son comparados entre sí, junto con resultados referenciales obtenidos mediante otros tres métodos que son: absorción atómica, diferencia de masa de las muestras antes y después de ser impregnadas (método gravimétrico) y cenizas resultantes de su calcinación (descontando las cenizas propias de la madera). También se realizan consideraciones respecto de la complejidad operacional de los distintos métodos implementados, con fines de comparación para la determinación rutinaria de la retención de arseniato de cobre cromatado en la madera.

\section{METODOLOGÍA}

A partir de una rodela obtenida a 1,3 m de altura de un híbrido experimental de 5 años de edad cuyos parentales eran Populus trichocarpa (Torr. \& Gray) y Populus deltoides (Bart. ex Marsh.) se obtuvo harina de madera utilizando un molino de cuchillos Retsch modelo SM-100. Se homogenizó su granulometría mediante tamices de 20 y 40 mesh. Esta harina de madera fue separada en 32 muestras homogéneas de alrededor de $30 \mathrm{~g}$ cada una y secaron en estufa hasta masa constante. Se utilizó preservante CCA-C comercial al 60 \% para preparar 32 soluciones diluidas de distinta concentración. Estas soluciones abarcaron un rango de concentración (gramos de preservante por litro de solución) que iba desde $2,49 \mathrm{~g} \mathrm{~L}^{-1}$ hasta $16,70 \mathrm{~g} \mathrm{~L}^{-1}$. Cada una de las muestras de madera anhidra fue puesta en un vaso precipitado tarado al cual se vertieron $180 \mathrm{~mL}$ de la solución CCA-C diluida preparada en el laboratorio. Estas muestras se dejaron reposar a temperatura ambiente por 24 horas. $\mathrm{Al}$ cabo de este tiempo, se puso cada vaso precipitado en estufa a una temperatura de $103^{\circ} \mathrm{C}$ hasta evaporar completamente la solución.

Se determinó la nueva masa de cada muestra descontando la masa del vaso (que había sido determinada previamente). Se estimó la retención expresada en $\mathrm{kg}$ óxidos activos $\mathrm{kg}^{-1}$ madera mediante el incremento de masa $(\mathrm{m})$ de cada muestra, usando la siguiente expresión:

$$
\text { Retención }=\frac{\left(m_{\text {muestra impregnada }}-m_{\text {muestra inicial }}\right)}{m_{\text {muestra inicial }}}
$$

Posteriormente, se extrajo alrededor de $2 \mathrm{~g}$ a partir de cada una de las 32 muestras y de otras 4 muestras de harina de madera que no había sido impregnadas y se calcinaron en una mufla a $575^{\circ} \mathrm{C}$ siguiendo el procedimiento indicado por Torres et al. (2011). Se determinó la masa de cenizas usando balanza analítica.

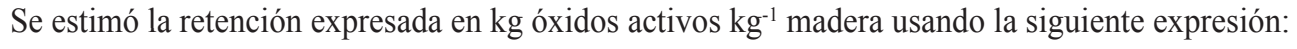

$$
\text { Retención }=\frac{(\text { m cenizas muestra impregnada }-m \text { cenizas muestra no impregnada })}{m_{\text {muestra inicial }}}
$$

Mediante la técnica de espectrofotometría de absorción atómica se determinó el contenido de cobre en las muestras de madera impregnadas con CCA-C. Las muestras fueron secadas en estufa con aire forzado a $70^{\circ} \mathrm{C}$, hasta peso constante. Posteriormente, fueron sometidas a molienda y tamizadas con malla de Nylon de $1,0 \mathrm{~mm}$ de tamaño. Para el tratamiento químico, se pesó $1,0 \mathrm{~g}$ de muestra tamizada en crisol de porcelana, se calcinó lentamente hasta alcanzar los $500^{\circ} \mathrm{C}$ durante 6 horas. Los crisoles se dejaron enfriar hasta temperatura ambiente, luego se adicionó bajo campana $2 \mathrm{~mL}$ de agua bidestilada y $10 \mathrm{~mL}$ de ácido clorhídrico 2 molar. Las soluciones se sometieron a calentamiento en placa calefactora bajo constante agitación, hasta casi sequedad. Posteriormente, las soluciones fueron filtradas utilizando filtros de $0,45 \mu \mathrm{m}$ de porosidad. El filtrado se llevó a un volumen final de $50 \mathrm{~mL}$ con agua bidestilada (Karla 1998, Walinga et al. 1995). Las determinaciones de cobre se realizaron por AA con técnica de llama (aire/acetileno), utilizando un espectrofotómetro Unicam modelo 969. El instrumento fue calibrado con soluciones estándar Fisher Scientific Internacional Company. Todas las determinaciones incluyeron sus respectivas soluciones blanco. La validación de la metodología se realizó utilizando material de referencia certificado SRM-1570 del National Institute of Standards and Technology (Guerra et al. 2009). El error relativo obtenido para cobre con 3 repeticiones fue $+8,6 \%$ con un coeficiente de variación de $1,92 \%$. 
El remanente de las muestras fue dejado al ambiente del laboratorio. La semana posterior a la determinación de retención, se extrajo alrededor de $2 \mathrm{~g}$ a partir de cada una de las 32 muestras de harina impregnada de madera. Cada una de ellas se puso en un portamuestra de plástico de forma cilíndrica de 23,5 $\mathrm{mm}$ de diámetro interno. En un extremo, soportando la muestra, se puso una lámina de Mylar de $10 \mu \mathrm{m}$ de espesor, tensada con un anillo externo. Cada muestra se comprimió utilizando una máquina de ensayo universal Instron modelo 3369. La velocidad de avance en compresión fue fijada en $2 \mathrm{~mm} \mathrm{~min}^{-1}$. La compresión de la muestra se detuvo a una tensión unitaria de 2,3 MPa, que permaneció durante 5 segundos. Se verificó la uniformidad del espesor y consistencia de las muestras. Enseguida cada una fue sometida a un proceso de irradiación mediante un tubo de X Röntgen-Technik Dr. Warrikhoff KG alimentado por un generador de alta tensión Spellman modelo XLG. Durante todo el procedimiento, la tensión, amperaje y duración del conteo de fotones fueron fijadas en 25 $\mathrm{Kv} ; 0,015 \mathrm{~mA}$ y 120 segundos, respectivamente. El haz incidente fue reducido a un diámetro de $10 \mathrm{~mm}$ justo antes de irradiar la muestra en un ángulo de $45^{\circ}$ respecto de su superficie. En un ángulo de $90^{\circ}$ respecto al haz incidente se instaló un detector espectrométrico de rayos X Amptek modelo XRG-100 que fue conectado a computador personal utilizando como interface un analizador multicanal modelo MCA8000A del mismo fabricante. También se realizó la irradiación de láminas de cobre y molidbeno de alta pureza (Goodfellow Cambridge Limited) con el propósito de calibrar la energía fotónica del analizador multicanal, resultando un valor $\mathrm{R}^{2}$ igual a 0,999 . Mediante una aplicación Visualbasic especialmente creada se determinaron las intensidades espectrales correspondientes a los picos $\mathrm{K} \alpha$ de cromo $(5,41 \mathrm{keV})$, cobre $(8,05 \mathrm{keV})$ y arsénico $(10,54 \mathrm{keV})$. Para ello se sumaron las intensidades de 5 canales equivalentes $0,1 \mathrm{keV}$ en torno a cada pico.

Las mediciones de reflectancia difusa de infrarrojos se realizaron en un analizador de reflectancia difusa Unity modelo 2200 que cubrió un rango espectral desde 1100 a $2200 \mathrm{~nm}$ con una resolución espacial de $1 \mathrm{~nm}$. Se utilizó el portamuestra proporcionado por el fabricante con las muestras sin realizar compresión alguna. El tiempo de escaneo por cada muestra alcanzó los 150 segundos, promediándose internamente por el dispositivo 120 mediciones espectrales realizadas en ese lapso. Se realizaron análisis de regresión por mínimos cuadrados parciales utilizando el programa Calstar 2.00 (Sensologic - Germany). No se realizó ningún tipo de pretratamiento de datos espectrales. Se seleccionaron aleatoriamente 20 muestras como subconjunto de calibración (aproximadamente 2/3 del total) y las restantes 12 pasaron a formar parte del subconjunto de validación. Calstar seleccionó automáticamente la cantidad de factores en base a un proceso de minimización del error estándar de validación cruzada (SECV). El output del programa, es decir, el listado de parejas "valor de referencia versus valor predicho" fue almacenado en una planilla de cálculo.

La calidad de todas las regresiones fue medida mediante el coeficiente de determinación $\left(\mathrm{R}^{2}\right)$, el cociente de performance respecto a la desviación (ratio prediction to deviation - RPD), el error absoluto promedio (average absolute error - $\mathrm{AAE} \%$ ), el error de sesgo promedio (average bias error - $\mathrm{ABE} \%$ ) y el porcentaje que representa la raíz del error cuadrático de la predicción respecto de la media (RMSE\%). Estos últimos fueron calculados de acuerdo a las ecuaciones $3,4,5$ y 6 .

$$
\begin{array}{r}
R P D=\left(1-R^{2}\right)^{-1 / 2} \\
A A E \%=\frac{1}{n} \sum_{i=1}^{n}\left|\frac{\hat{X} i-X i}{X i}\right| \times 100 \% \\
A B E \%=\frac{1}{n} \sum_{i=1}^{n} \frac{(\hat{X} i-X i)}{X i} \times 100 \% \\
R M S E \%=\frac{\sqrt{\sum[(X i-\hat{X} i)]^{2} / n}}{\bar{X}} \times 100 \%
\end{array}
$$


Cabe indicar que un $\mathrm{AAE} \%$ bajo indica un pequeño error de correlación, mientras que un $\mathrm{ABE}$ positivo implica un sesgo de sobreestimación y un ABE\% negativo indica un sesgo de subestimación (Sheng y Azevedo 2005). Por otra parte, Williams (2001) sugiere que valores RPD entre 3,0 y 5,0 son aceptables para fines predictivos, mientras que valores entre 5,0 y 8,0 son adecuados con propósitos de control rutinario de calidad y valores superiores a 8,0 deben ser considerados como excelentes.

\section{RESULTADOS Y DISCUSIÓN}

La Figura 1 presenta tres espectros de fluorescencia de rayos $\mathrm{X}$ obtenidos para la muestra de mayor retención (espectro más intenso), menor retención (espectro menos intenso) y para una muestra de retención intermedia. Se aprecia en torno a 5,41 y 5,95 keV las líneas de emisión $\mathrm{K} \alpha$ y $\mathrm{K} \beta$ de cromo, respectivamente. En torno a 8,05 y $8,90 \mathrm{keV}$ aparecen las líneas de emisión de cobre y en torno a 10,54 y 11,73 keV las que corresponden a arsénico. En torno a 9,67 keV aparece un pico que coincide con la línea $\mathrm{L} \beta$ del tungsteno que es propio del anticátodo del tubo de rayos X. Otro pico a $8,40 \mathrm{keV}$ correspondiente a la línea L $\alpha$ del tungsteno no está resuelto por el detector y aparece superpuesto a la línea Ka de cobre. La Figura 2 presenta los mismos tres espectros de reflectancia difusa de infrarrojos cercanos de la Figura 1.

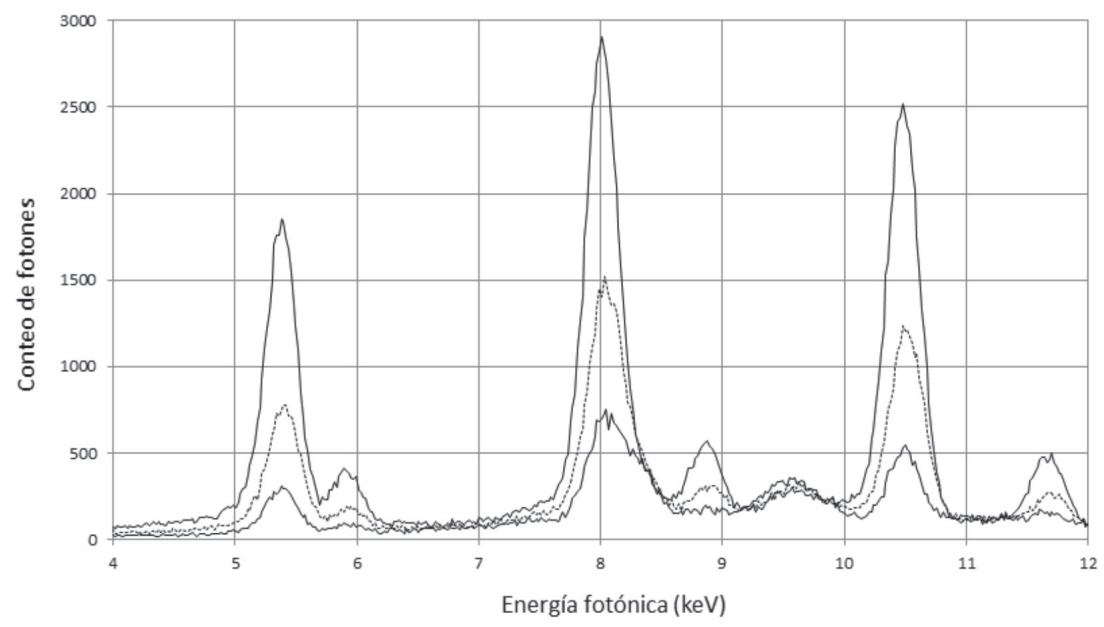

Figura 1. Espectros de fluorescencia registrados en un periodo de 120 segundos.

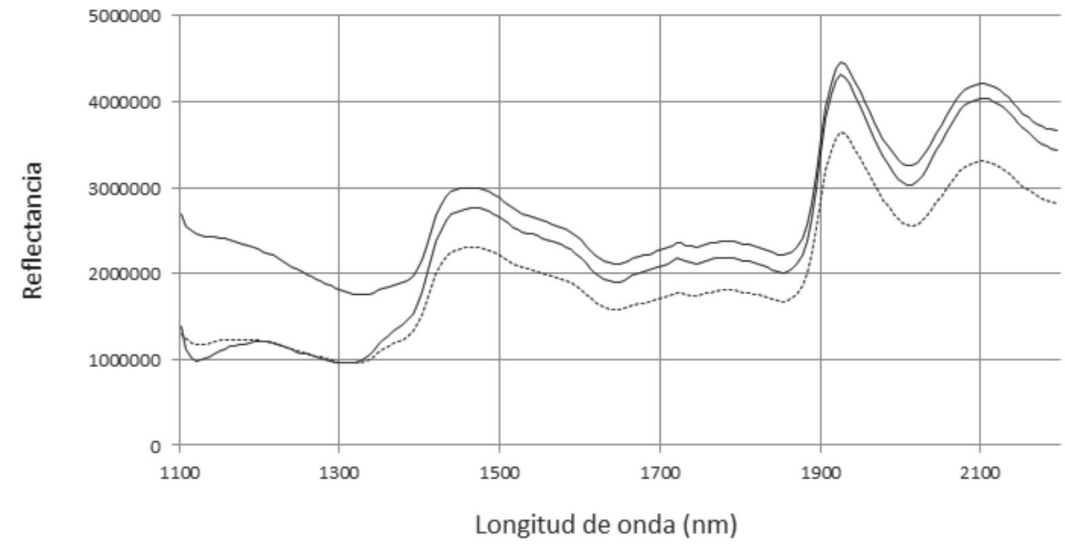

Figura 2. Espectros de reflectancia difusa de infrarrojos registrados en un periodo de 150 segundos. 
Un ensayo de muestras pareadas entre la retención por método gravimétrico y la retención por cenizas, para el conjunto de 32 determinaciones, indica que existen diferencias significativas entre ambos métodos (valor- $\mathrm{p}=0,0001$ ). De modo que la retención por cenizas es $13,2 \%$ inferior a la retención por diferencia de masa, con un coeficiente $\mathrm{R}^{2}$ igual a 0,985 . Por otra parte, las concentraciones de óxido de cobre determinadas por AA resultan en proporciones de $14,4 \%\left(\mathrm{R}^{2}=0,932\right)$ y $16,7 \%\left(\mathrm{R}^{2}=0,951\right)$ de la retención (total) medida por método gravimétrico y de cenizas, respectivamente. Sin embargo, esta proporción es de $18,5 \%$ en la solución comercial de CCA-C utilizada en el estudio.

Los gráficos de dispersión y los valores $\mathrm{R}^{2}$ y RPD para todas las combinaciones de análisis de regresión se presentan en Figura 3 y en las Tablas 1 y 2 . En todos los casos, estos subconjuntos estuvieron compuestos por las mismas muestras elegidas de forma aleatoria. Las calibraciones en que está presente el espectro NIR fueron obtenidas mediante PLSR sin pretratamiento espectral, resultando a partir de un modelo con 3 factores. Las curvas de regresión entre XRF y los otros métodos siguen curvas potenciales $\left(Y=a X^{b}\right)$ obtenidas por mínimos cuadrados simples. Para el resto se obtuvieron expresiones lineales $(\mathrm{Y}=\mathrm{a}+\mathrm{b} \mathrm{X})$ mediante mínimos cuadrados simples.

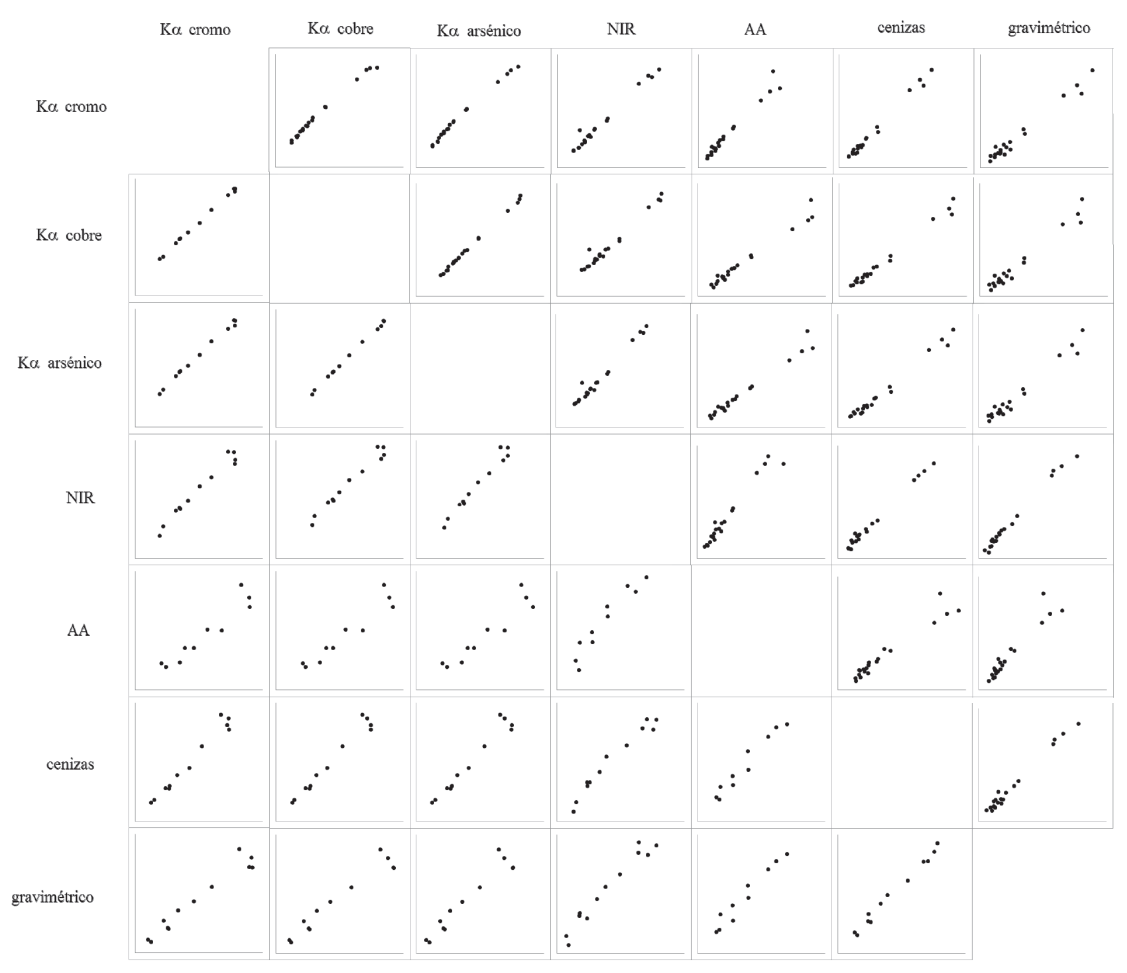

Figura 3. Gráficos de dispersión para el conjunto de calibración (parte alta) y de predicción (parte baja). 
Tabla 1. Resumen de valores de $\mathrm{R}^{2}$ en conjunto de calibración (parte alta) y de predicción (parte baja).

\begin{tabular}{|c|c|c|c|c|c|c|c|}
\hline & Ka cromo & $\begin{array}{c}\text { XRF } \\
\text { Ka cobre }\end{array}$ & Ka arsénico & NIR & $\mathbf{A A}$ & cenizas & masa \\
\hline Ka cromo & - & 0,993 & 0,994 & 0,980 & 0,969 & 0,976 & 0,923 \\
\hline Ka cobre & 0,998 & - & 0,998 & 0,973 & 0,966 & 0,973 & 0,904 \\
\hline Ka arsénico & 0,995 & 0,999 & - & 0,975 & 0,972 & 0,966 & 0,910 \\
\hline NIR & 0,970 & 0,974 & 0,974 & - & 0,957 & 0,991 & 0,990 \\
\hline $\mathbf{A A}$ & 0,920 & 0,929 & 0,909 & 0,918 & - & 0,94 & 0,915 \\
\hline cenizas & 0,986 & 0,986 & 0,981 & 0,962 & 0,952 & - & 0,984 \\
\hline masa & 0,953 & 0,951 & 0,945 & 0,953 & 0,948 & 0,988 & - \\
\hline
\end{tabular}

Tabla 2. Resumen de valores de RPD en conjunto de calibración (parte alta) y de predicción (parte baja).

\begin{tabular}{|c|c|c|c|c|c|c|c|}
\hline & K $\alpha$ cromo & $\begin{array}{c}\text { XRF } \\
\text { Ka cobre }\end{array}$ & K $\alpha$ arsénico & NIR & $\mathbf{A A}$ & cenizas & masa \\
\hline Ka cromo & - & $12,0^{\mathrm{e}}$ & $12,9^{\mathrm{e}}$ & $7,1^{b}$ & $5,7^{\mathrm{b}}$ & $6,5^{\mathrm{b}}$ & $3,6^{\mathrm{a}}$ \\
\hline Ka cobre & $22,4^{\mathrm{e}}$ & - & $22,4^{\mathrm{e}}$ & $6,1^{\mathrm{b}}$ & $5,4^{\mathrm{b}}$ & $6,1^{\mathrm{b}}$ & $3,2^{\mathrm{a}}$ \\
\hline Ka arsénico & $14,1^{\mathrm{e}}$ & $31,6^{\mathrm{e}}$ & - & $6,3^{\mathrm{b}}$ & $6,0^{\mathrm{b}}$ & $5,4^{\mathrm{b}}$ & $3,3^{a}$ \\
\hline NIR & $5,8^{\mathrm{b}}$ & $6,2^{\mathrm{b}}$ & $6,2^{\mathrm{b}}$ & - & $4,8^{\mathrm{a}}$ & $10,5^{\mathrm{e}}$ & $10,0^{\mathrm{e}}$ \\
\hline AA & $3,5^{\mathrm{a}}$ & $3,8^{\mathrm{a}}$ & $3,3^{\mathrm{a}}$ & $3,5^{\mathrm{a}}$ & - & $4,1^{\mathrm{a}}$ & $3,4^{\mathrm{a}}$ \\
\hline cenizas & $8,5^{\mathrm{e}}$ & $8,5^{\mathrm{e}}$ & $7,3^{\mathrm{b}}$ & $5,1^{\mathrm{b}}$ & $4,6^{\mathrm{a}}$ & - & $7,9^{\mathrm{b}}$ \\
\hline masa & $4,6^{\mathrm{a}}$ & $4,5^{\mathrm{a}}$ & $4,3^{\mathrm{a}}$ & $4,6^{\mathrm{a}}$ & $4,4^{\mathrm{a}}$ & $9,1^{\mathrm{e}}$ & - \\
\hline
\end{tabular}

En todos los casos el valor $\mathrm{R}^{2}$ es superior a $0,909 \mathrm{y}$ todas las regresiones resultaron altamente significativas. Del análisis de los valores RPD se aprecia que en todos los casos se obtienen resultados superiores a 3,0, es decir, que son aceptables para fines predictivos.

Debido a que los valores $\mathrm{R}^{2}$ y RPD por sí solos no dan cuenta completa de la calidad predictiva de la curva de calibración, se presentan los valores del error de sesgo promedio (ABE\%) y el error absoluto promedio (AAE\%) (Tabla 3) y el porcentaje que representa la raíz del error cuadrático de la predicción respecto de la media RMSEC\% o RMSEP\% en el subconjunto de calibración y en el subconjunto de predicción, respectivamente (Tabla 4).

Tabla 3. Resumen de valores de $\mathrm{ABE} \%$ / $\mathrm{AAE} \%$ en conjunto de calibración (parte alta) y de predicción (parte baja).

\begin{tabular}{cccccccc}
\hline & $\mathbf{K} \boldsymbol{\alpha}$ cromo & $\begin{array}{c}\text { Fluorescencia } \\
\mathbf{K} \boldsymbol{\alpha} \text { cobre }\end{array}$ & $\mathbf{K} \boldsymbol{\alpha}$ arsénico & NIR & AA & cenizas & masa \\
\hline Ko cromo & - & $0,6 / 3,4$ & $0,9 / 4,3$ & $0,9 / 7,0$ & $0,6 / 9,2$ & $0,6 / 8,8$ & $2,2 / 16,1$ \\
$\mathbf{K} \boldsymbol{\alpha}$ cobre & $0,1 / 1,4$ & - & $0,2 / 2,1$ & $1,1 / 5,8$ & $0,7 / 9,0$ & $0,4 / 8,9$ & $2,5 / 18,5$ \\
$\mathbf{K} \boldsymbol{\alpha}$ arsénico & $0,3 / 2,3$ & $0,1 / 1,3$ & - & $1,3 / 6,7$ & $0,5 / 8,4$ & $0,8 / 10,5$ & $2,5 / 18,3$ \\
NIR & $2,7 / 7,3$ & $1,9 / 5,2$ & $2,0 / 4,9$ & - & $0,7 / 10,4$ & $0,5 / 10,6$ & $-0,2 / 10,7$ \\
AA & $1,1 / 12,6$ & $0,9 / 12,1$ & $1,3 / 13,6$ & $2,4 / 15,6$ & - & $4,4 / 14,3$ & $5,3 / 15,9$ \\
cenizas & $0,3 / 5,7$ & $0,1 / 6,1$ & $0,4 / 7,1$ & $-0,3 / 12,4$ & $1,2 / 11,7$ & - & $2,0 / 12,6$ \\
masa & $1,4 / 14,3$ & $0,5 / 14,9$ & $3,3 / 16,3$ & $10,3 / 21,1$ & $1,2 / 12,9$ & $0,1 / 7,5$ & - \\
\hline
\end{tabular}


Tabla 4. Resumen de valores de RMSEC\% (parte alta) y RMSEP\% (parte baja).

\begin{tabular}{|c|c|c|c|c|c|c|c|}
\hline & & Fluorescencia & & NIR & AA & cenizas & masa \\
\hline & Ka cromo & K $\alpha$ cobre & Ka arsénico & & & & \\
\hline Ka cromo & - & 3,9 & 4,1 & 8,8 & 13,4 & 8,7 & 14,4 \\
\hline Ka cobre & 1,5 & - & 2,2 & 8,0 & 11,5 & 10,4 & 17,8 \\
\hline Ka arsénico & 2,4 & 1,4 & - & 8,3 & 11,9 & 12,0 & 18,0 \\
\hline NIR & 7,8 & 6,0 & 6,5 & - & 15,3 & 7,7 & 8,5 \\
\hline $\mathbf{A A}$ & 14,2 & 13,9 & 15,3 & 14,4 & - & 18,1 & 21,5 \\
\hline cenizas & 9,3 & 9,6 & 11,1 & 10,6 & 10,0 & - & 10,2 \\
\hline masa & 14,5 & 15,7 & 17,1 & 14,5 & 10,3 & 5,8 & - \\
\hline
\end{tabular}

Para el conjunto de calibración los valores $\mathrm{ABE} \%$, AAE\% y RMSEC\% más altos (calibraciones menos robustas) se encuentran para el método de AA relacionado con el contenido de cenizas y con el aumento de masa y para la fluorescencia de cobre y arsénico relacionada con el aumento de masa. Para el conjunto de predicción los valores $\mathrm{ABE} \%, \mathrm{AAE} \%$ y RMSEP\% más altos se encuentran para la fluorescencia de cobre y arsénico relacionada con el aumento de masa y para el método NIR relacionado con el aumento de masa. Los valores mejores se obtienen vinculando los resultados de fluorescencia entre sí y vinculando estos con la cantidad de cenizas resultantes de la calcinación.

De todos los métodos implementados en este trabajo, sólo el gravimétrico sería capaz de entregar un valor real de la retención de óxidos activos en muestras de madera impregnada con CCA. El método de cenizas resultantes de calcinación también podría entregar un valor real de la retención de óxidos, suponiendo que durante la calcinación sólo una pequeña parte de cobre, cromo y arsénico pasan al aire y la mayor parte de ellos quedase como cenizas. Sin embargo, se indica que entre $13 \%$ y $27 \%$ y entre $22 \%$ y $44 \%$ del arsénico es volatilizado a temperaturas de combustión de $400^{\circ} \mathrm{C}$ y $800^{\circ} \mathrm{C}$, respectivamente (McMahon et al. 1986), por lo cual el supuesto pudiera no ser válido. A su vez, el método de AA utilizado entrega sólo el valor de la concentración o retención de óxido de cobre y no del total de óxidos. Finalmente, los métodos XRF y NIR no son capaces de entregar un valor real de la retención de óxidos, aunque permiten obtener datos cuantitativos que se correlacionan favorablemente con ella.

La proximidad entre el resultado gravimétrico y el resultado de cenizas (13,2 \% de subestimación promedio) indica que las pérdidas de cromo, cobre y arsénico por volatilización fueron relativamente reducidas. De modo que el método por calcinación podría ser considerado adecuado para determinar la retención de óxidos en la madera impregnada con CCA. Sin embargo, los resultados de las calibraciones de XRF, NIRS y AA con respecto al método gravimétrico en todos los casos resultaron en menores valores $\mathrm{R}^{2}$ y mayores errores de predicción que las realizadas usando las cenizas como referencia. Esto evidencia el método gravimétrico presentado en este artículo no permitió obtener un nivel de precisión acorde con lo esperable para el que debía corresponder al método de referencia de los otros. Esta imprecisión se debió a la dificultad para determinar los aumentos de masa provocados por la fijación de CCA al impregnar pequeñas cantidades de madera (alrededor de $30 \mathrm{~g}$ ).

Por otra parte, los resultados demuestran que la determinación mediante AA de la concentración de óxido de cobre bastaría por sí sola para estimar la retención del total de óxidos activos. Esto simplificaría notoriamente la determinación de la retención total debido a que haría innecesarias las mediciones de cromo y arsénico, que son complejas de llevar a cabo.

Block et al. (2007) habían determinado que la mejor expresión vinculando la intensidad de fluorescencia del arsénico con la concentración de arsénico medida por AA seguía la curva $0,26 \times \mathrm{XRF}^{1,12}$. Esta expresión de tipo potencial es característica cuando se presenta absorción de la radiación fluorescente dentro de la matriz lignocelulósica impregnada. En caso de producirse en la matriz un efecto potenciador se obtendría una expresión logarítmica tipo $\alpha \mathrm{x} \log (\mathrm{XRF})+b$. En este estudio, todas las curvas de calibración de XRF siguieron una expresión potencial cuya robustez según los criterios descritos más arriba varió desde aceptable para fines predictivos $(\mathrm{RPD}=3,3)$ hasta excelente $(\mathrm{RPD}=10,5)$. De hecho, los valores $\mathrm{R}^{2}$ y $\mathrm{RPD}$ del método 
XRF ya sea para las líneas K $\alpha$ de cromo, cobre o arsénico estuvieron sistemáticamente entre los más altos. En particular, el método XRF tanto de cromo, cobre y arsénico con respecto a la retención medida por cenizas logra valores RPD considerados como buenos para propósitos de control de calidad o incluso excelentes. Así mismo, los valores $\mathrm{R}^{2}$ no se deterioran al aplicar las curvas de calibración en el conjunto de predicción, lo cual, da cuenta de la robustez de la capacidad predictiva de las calibraciones XRF.

En el caso de NIR las curvas de calibración obtenidas entregan resultados superiores a los obtenidos por So et al. (2004) quienes obtuvieron valores RMSEP\% de 23,9;20,6 y 16,7\% para las concentraciones de pentóxido de arsénico, óxido de cromo y óxido de cobre, respectivamente. Estos resultados son también superiores a los presentados por So et al. (2006) cuya calibración presentó un valor $\mathrm{R}^{2}$ de 0,94 y RMSEC\% de $18,0 \%$ en una muestra compuesta por 14 datos. Así mismo, la calidad de las calibraciones es superior a la presentada por Taylor y Lloyd (2007) que para la determinación de la retención de octoborato de sodio determinaron un valor de $\mathrm{R}^{2}$ igual a 0,96 en el conjunto de calibración compuesto por 18 datos que se redujo a un $\mathrm{R}^{2}$ igual a 0,86 (RMSEP\% cercano a $25 \%$ ) en el subconjunto de predicción conformado por otras 10 muestras. Pese a estos buenos resultados, debe tomarse en cuenta que en el presente estudio los valores $\mathrm{R}^{2}$ y RPD se redujeron en el conjunto de predicción con respecto a los obtenidos en el conjunto de calibración. Aun así, los valores RPD siguen siendo aceptables para fines predictivos, pues fueron superiores a 3,0 en todos los casos.

En general, los resultados menos robustos se obtuvieron cuando estuvieron involucrados los métodos que a priori podían haber sido considerados como los mejores, como son el método AA y el método gravimétrico.

Respecto al nivel de simplicidad operacional de las mediciones de CCA a partir de harina tamizada de madera impregnada, puede indicarse que el método que requiere una menor cantidad de recursos es el de determinación de cenizas, para lo cual, sólo se requiere de mufla y balanza analítica. Operacionalmente el método NIRS resulta ser simple pues sólo se deposita una cantidad similar de harina de madera al interior del portamuestra del dispositivo, sin controlar la densificación de ella y el analizador rápidamente realiza unas lecturas de reflectancia difusa bien correlacionadas con la retención. Sin embargo, se requiere de una etapa de obtención de calibraciones usando análisis multivariado para gestionar la relación compleja entre los espectros registrados y las concentraciones de óxidos. El método XRF también es de rápida ejecución y entrega de forma simultánea tres nítidos picos espectrales que se correlacionan directamente con la concentración de los óxidos respectivos. Aunque este método requiere tomar mayores precauciones respecto al nivel de densificación de las muestras pues, en resultados no presentados aquí, se obtuvo que para una misma muestra de madera impregnada, la intensidad de fluorescencia $\mathrm{K} \alpha$ de cobre y de arsénico aumentó a medida que aumentó la densificación de la muestra. Aunque esta intensidad se mantuvo constante para la emisión fluorescente $\mathrm{K} \alpha$ de cromo en un amplio rango de densificación. Por otra parte, este método añade la complejidad de manipular un dispositivo que genera radiaciones ionizantes. En comparación con los otros métodos, el de absorción atómica es más complejo y lento de ejecutar, por lo cual, parece poco adecuado si se dispone de las otras alternativas.

\section{CONCLUSIONES}

Los resultados $\mathrm{R}^{2}$ de análisis de correlación entre la intensidad de fluorescencia de las líneas $\mathrm{K} \alpha$ de cromo, cobre y arsénico con respecto a la retención de CCA medida por cenizas resultantes de calcinación, absorción atómica y diferencia de masa antes y después de impregnación son altos. El valor $\mathrm{R}^{2}$ se encuentra en un rango de 0,909 a 0,986 y el valor RPD se encuentra en un rango de 3,3 a 8,5 tanto en un conjunto de muestras usado como calibración, en que se desarrolla el análisis de regresión, como en un conjunto independiente de predicción o verificación. 
Los resultados $\mathrm{R}^{2}$ de análisis de correlación entre el espectro de reflectancia difusa infrarroja con respecto a la retención de CCA medida por cenizas resultantes de calcinación, absorción atómica y diferencia de masa antes y después de impregnación son altos. El valor $\mathrm{R}^{2}$ se encuentra en un rango de 0,918 a 0,962 y el valor RPD se encuentra en un rango de 3,5 a 5,1 para el conjunto de muestras de predicción. Estos resultados son de menor robustez a los obtenidos por fluorescencia de rayos $\mathrm{X}$.

\section{AGRADECIMIENTOS}

Los investigadores agradecen a la empresa Comercial Osmose Chile Limitada por proporcionar a título gratuito el preservante CCA-C utilizado en la ejecución del estudio.

\section{BIBLIOGRAFÍA}

AWPA. 1990. Standard method for analysis of treated wood and treating solutions by $x$-ray spectroscopy (A9-90). American wood-preservers' association standard (AWPA).

AWPA. 1991. Standard for Waterborne Preservatives (P5-91). American wood-preservers' association standard (AWPA).

Baettig, R.; Perré, P.; Rémond, R. 2007. Aplicación de espectrometría de rayos X para el estudio de la madera: Algunos resultados iniciales. Maderas. Ciencia y tecnología 9(3): 271-283.

Block, C.; Shibata, T.; Solo-Gabriele, H.; Townsend, T. 2007. Use of handheld X-ray fluorescence spectrometry units for identification of arsenic in treated wood. Environmental Pollution 148: 627 - 633.

Brunet, D.; Barthès, B.; Chotte, J.; Feller, C. 2007. Determination of carbon and nitrogen contents in Alfisols, Oxisols and Ultisols from Africa and Brazil using NIRS analysis: Effects of sample grinding and set heterogeneity. Geoderma 139: 106 - 117.

Chang, C.; Laird, D.; Mausbach, M.; Hurburgh, C. 2001. Near-Infrared Reflectance SpectroscopyPrincipal Components Regression Analyses of Soil Properties. Soil Science Society of America Journal 65: $480-490$.

Fengel, D.; Wegener, G. 1984. Wood: Chemistry, Ultrastructure, Reactions. W. de Gruyter. 613p.

Gaspar, F.; Lopes, J.; Cruz, H.; Schwanninger, M.; Rodrigues, J. 2009. Application of near infrared spectroscopy and multivariate data analysis for the evaluation of glue lines of untreated and copper azole treated laminated timber before and after ageing. Polymer Degradation and Stability 94(7): 1061-1071.

Guerra, F.; Duplessis, S.; Kohler, A.; Martin, F.; Tapia, J.; Lebed, P.; Zamudio, F.; González, E. 2009. Gene expression analysis of Populus deltoides roots subjected to copper stress. Environmental and Experimental Botany 67: 335- 344.

Hein, P.; Campos, A.; Mendes, R.; Mendes, L.; Chaix, G. 2011. Estimation of physical and mechanical properties of agro-based particleboards by near infrared spectroscopy. European Journal of Wood and Wood Products 69(3): 431-442.

Karla, Y.P. 1998. Handbook of reference methods for plant analysis. Soil and Plant Analysis Council, Inc. CRC Press, USA. 300p. 
Krishna, A.; Mohan, K.; Satyanarayanan, M. 2012. Non-destructive Sampling Method for Environmental and Geochemical Exploration Studies and XRF Determination of $\mathrm{Cl}, \mathrm{F}$, and $\mathrm{Br}$. Atomic Spectroscopy 33(3): 100-108.

McMahon, Ch.; Bush, P.; Woolson, E. 1986. How much arsenic is released when CCA treated wood is burned?. Forest Products Journal 36(11-12): 45 - 50.

Osborne, B. 2006. Near-infrared Spectroscopy in Food Analysis. Encyclopedia of Analytical Chemistry. Wiley Online Library.

Qi, J.L.; Qi, J.R.; Wang, Z.; Yang, G.; Wang, C. 2013. Determination of Cobalt in Fertilizer by Wavelength Dispersive X-Ray Fluorescence Spectrometry. Advanced Materials Research 781: 2336-2339.

Sheng, C.; Azevedo, J. 2005. Estimating the higher heating value of biomass fuels from basic analysis data. Biomass and Bioenergy 28: 499-507.

Skoog, D.; Holler, F.; Nieman, T. 2001. Principios de Análisis Instrumental. Quinta edición. Mc Graw Hill Interamericana. Madrid. 997p.

So, C.; Lebow, S.; Groom, L.; Rials, T. 2004. The application of near infrared (NIR) spectroscopy to Inorganic preservative-treated wood. Wood and Fiber Science 36(3): 329 - 336.

So, C.; Eberhardt, T.; Lebow, S.; Groom, L. 2006. A Preliminary Study of Preservative Retention and Penetration in ACQ-Treated Timbers Using Near Infrared Spectroscopy. Seminar Wood Protection 2006. March 21-23 - 2006.

Taylor, A.; Lloyd, J. 2007. Potential of near infrared spectroscopy to quantify boron concentration in treated wood. Forest Products Journal 57(1-2): 116 - 117.

Torres, M.; Figueroa, V.; Vives, I. 2011. Biodeterioro en rolletes de Pinus radiata preservados con sal CCA frente al ataque de hongos de pudrición parda. Bosque 32(3): 315 - 320.

Walinga, I.; Van der Lee, J.; Houba, V.J.G.; Van Vark, W.; Novozamsky, I. 1995. Plant analysis manual. Kluwer Academia Publishers, Dordrecht, The Netherlands, 253p.

Welz, B.; Sperling, M. 1999. Atomic Absorption Spectrometry. Third Completely Revised. Edition. Wiley-VCH. Weinheim. Federal Republic of Germany. 1147 pp.

Williams, P. 2001. Implementation of near infrared technology. In Williams, P., Norris, K. (eds.) Near infrared technology in the agricultural and food industries. American Association of Cereal Chemist. St. Paul, Minnesota, USA. p. 145-169

Zielenkiewicz,T.; Zawadzki, J.; Radomski, A. 2012. XRF spectrometer calibration for copper determination in wood. X-Ray Spectrometry 41: 371- 373. 